\title{
Epstein-Barr Virus-associated Early Gastric Cancer Treated with Endoscopic Submucosal Dissection: A Possible Candidate for Extended Criteria of Endoscopic Submucosal Dissection
}

\author{
Hideo Yanai ${ }^{1}$, Daisuke Chihara ${ }^{1}$, Megumi Harano ${ }^{1}$, Eiki Sakaguchi ${ }^{1}$, \\ Tomoyuki Murakami ${ }^{2}$ and Jun Nishikawa ${ }^{3}$
}

\begin{abstract}
:
A 73-year-old man visited our hospital for the treatment of an early gastric cancer (GC) lesion. We performed en bloc resection using endoscopic submucosal dissection (ESD) for his GC lesion. The present GC lesion was Epstein-Barr virus (EBV)-associated poorly differentiated-type adenocarcinoma invading into the submucosal layer. Recently, accumulating data has shown that the risk of lymph node metastasis from early EBV GC without local lymphovascular infiltration is low. The present patient has been in good health for over three years since ESD. Some cases of early EBV GC with invasion into the submucosal layer may be candidates for further extension of the ESD criteria.
\end{abstract}

Key words: Epstein-Barr virus-associated gastric cancer, endoscopic submucosal dissection

(Intern Med 58: 3247-3250, 2019)

(DOI: 10.2169/internalmedicine.3055-19)

\section{Introduction}

Epstein-Barr virus (EBV) association is detected in almost $10 \%$ of gastric cancer (GC) lesions. EBV-associated GC (EBV GC) is a distinct subtype of GC with extreme DNA hypermethylation. Many EBV GCs are located in the upper part of the stomach and remnant stomach. EBV GC is mainly poorly differentiated, tumor-infiltrating lymphocyterich adenocarcinoma [carcinoma with lymphoid stroma (CLS)] (1-4). The treatment outcome of advanced EBV GC is reported to be better than that of EBV-negative GC. The risk for lymph node metastasis (LNM) of early EBV GC is also reported to be lower than that for EBV-negative early GC (5-7).

Currently, less-invasive endoscopic submucosal dissection (ESD) is widely used for early GC worldwide (8). However, poorly differentiated GC lesions with invasion into the submucosal layer are an indication for surgical operation with lymph node dissection because of the risk of LNM (9). Recently, further extension of the criteria for ESD for early GC has been suggested (10). EBV GC is a distinct subtype with a low risk of LNM. Therefore, further extension of the criteria for early EBV GC should be discussed.

We herein report a case of early EBV GC treated with ESD and discuss the further extension of the ESD criteria.

\section{Case Report}

A 73-year-old man visited our hospital for the further examination and treatment of his second early GC lesion. His initial GC lesion had been a differentiated-type intramucosal one and had been curatively resected at another hospital by endoscopic mucosal resection. He had been Helicobacter pylori-positive but received eradication therapy at his previous hospital.

Seven months after endoscopic resection, another GC lesion was detected by follow-up esophagogastroduodeno-

\footnotetext{
${ }^{1}$ Department of Clinical Research, Department of Gastroenterology \& Hepatology, National Hospital Organization Kanmon Medical Center, Japan, ${ }^{2}$ Department of Pathology, National Hospital Organization Kanmon Medical Center, Japan and ${ }^{3}$ Department of Laboratory Science, Yamaguchi University Graduate School of Medicine, Japan
}

Received for publication March 22, 2019; Accepted for publication June 12, 2019

Correspondence to Dr. Hideo Yanai, yanai.hideo.mu@mail.hosp.go.jp 


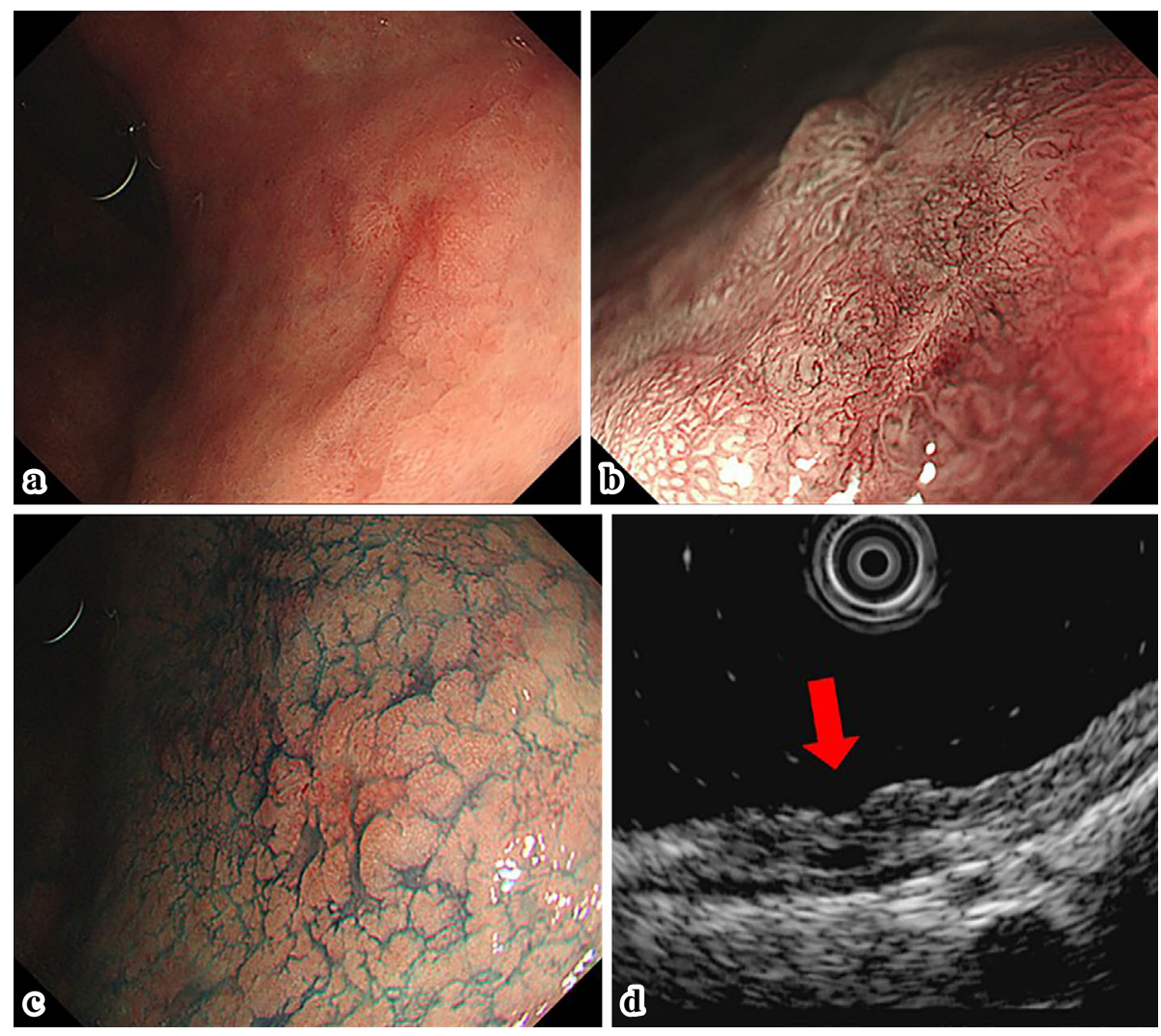

Figure 1. Endoscopic features of the early EBV GC lesion. a: White-light imaging observation. A superficial depressed-type early gastric cancer lesion was detected in the middle of the gastric body. The diameter of the lesion was almost $10 \mathrm{~mm}$. b: Narrow-band imaging revealed the irregular microvascular pattern of the lesion. c: Indigo-carmine dye contrast observation. A shallow, reddish depressed lesion was clearly visible. These ordinary endoscopic observations detected no signs of submucosal invasion, and the lesion was endoscopically staged as intramucosal cancer. d: However, thin-probe endoscopic ultrasonography detected a small hypoechoic mass less than $1 \mathrm{~mm}$ in diameter in the hyperechoic third layer, corresponding to the submucosal layer of the gastric wall, which was staged as being borderline invasion between the intramucosal layer and invading into the submucosa.

scopy. This second GC lesion was superficial depressed-type without ulcerous changes, $10 \mathrm{~mm}$ in diameter, and located in the middle gastric body. A gastric biopsy detected undifferentiated-type adenocarcinoma. He was referred to our hospital for possible endoscopic treatment using ESD based on the extended criteria for undifferentiated-type intramucosal adenocarcinoma $\leq 20 \mathrm{~mm}$ in diameter without ulcerous changes.

Abdominal computed tomography (CT) detected no lymph node swelling or metastatic lesion. Ordinary endoscopic observation detected no signs of invasion into the submucosal layer. Thin-probe endoscopic ultrasonography detected a small hypoechoic mass less than $1 \mathrm{~mm}$ in diameter in the hyperechoic third layer corresponding to the submucosal layer of the gastric wall, and it was staged as being at the borderline invasion stage between intramucosal and invading into the submucosal layer (11). Given these findings on the pretherapeutic examination, his second GC lesion was evaluated as a candidate for the extended criteria of ESD (Fig. 1).

We therefore performed en bloc resection using ESD for his second GC lesion (Fig. 2). The resection margin was tumor-free. The tumor was $10 \mathrm{~mm}$ in diameter with an invasion depth into the submucosal layer of $0.8 \mathrm{~mm}$.

The pathological type was CLS, and lymphovascular infiltration was negative according to D2-40 immune-staining and Elastica van Gieson (EVG) staining. A portion of the lesion was differentiated-type adenocarcinoma. Cancer lesions of mixed histological type in the stomach carry a risk of LNM (12). However, early CLS has distinct clinicopathological features, depending on the EBV infection status and LNM status (13). CLS had the characteristic histologic type of EBV GC, so we performed EBV-encoded small RNA1 in situ hybridization (EBER1 ISH) for the ESD specimen. Almost all tumor cell nuclei were EBER1positive, and his second GC lesion was confirmed to be EBV GC (Fig. 3). Retrospectively, his former GC lesion was found to be EBER1-negative.

The present EBV GC lesion was poorly differentiatedtype adenocarcinoma invading into the submucosal layer and evaluated as not meeting the extended criteria for ESD in the Japanese Gastric Cancer Treatment Guidelines (9). Be- 

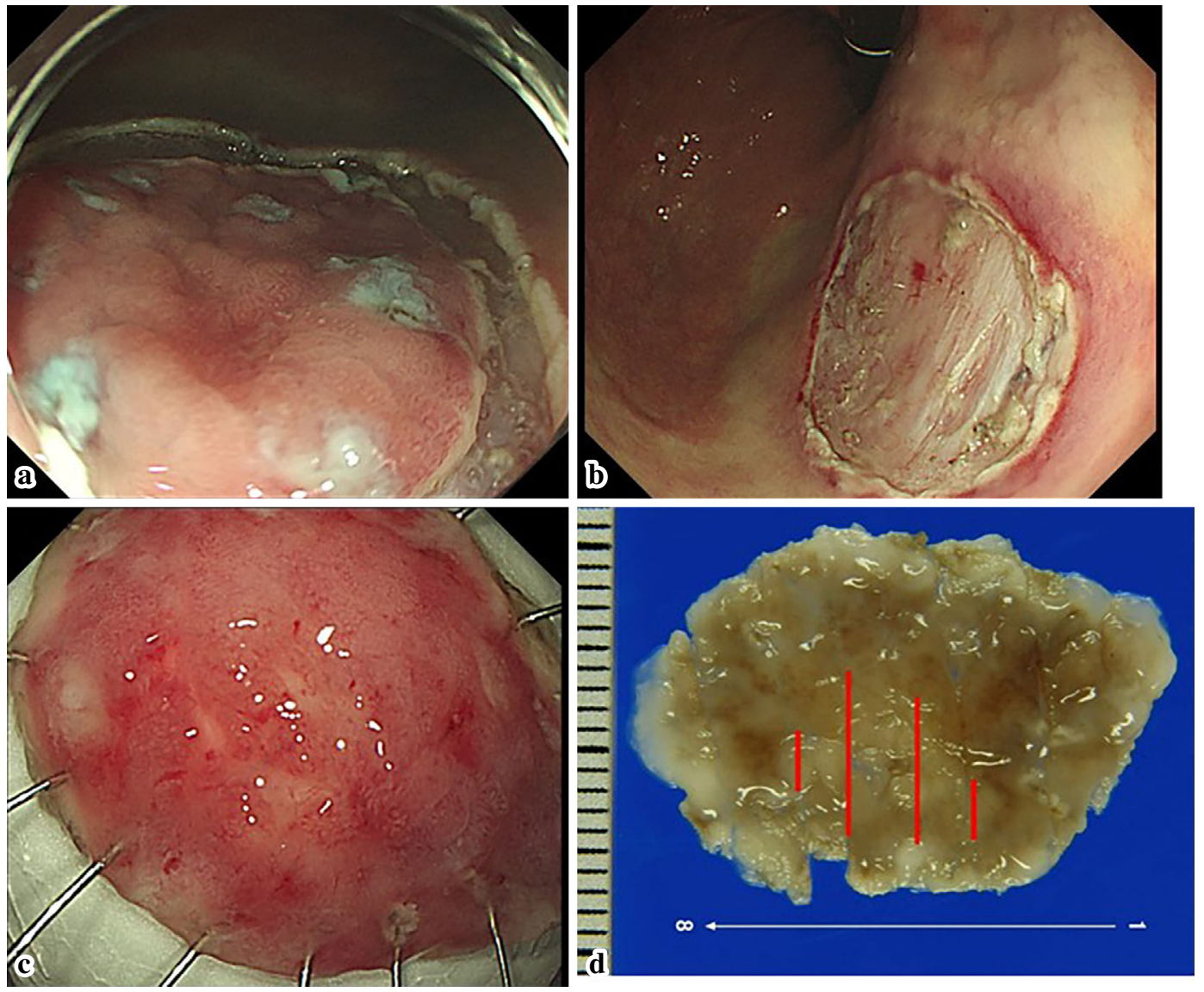

Figure 2. ESD for the early EBV GC lesion. Based on the results of the pretherapeutic examination, his GC lesion was considered a candidate for the extended criteria of ESD. a: ESD performance. b: There was no residual tumor after en bloc resection. c: ESD specimen. d: The tumor was located in the red-lined area.

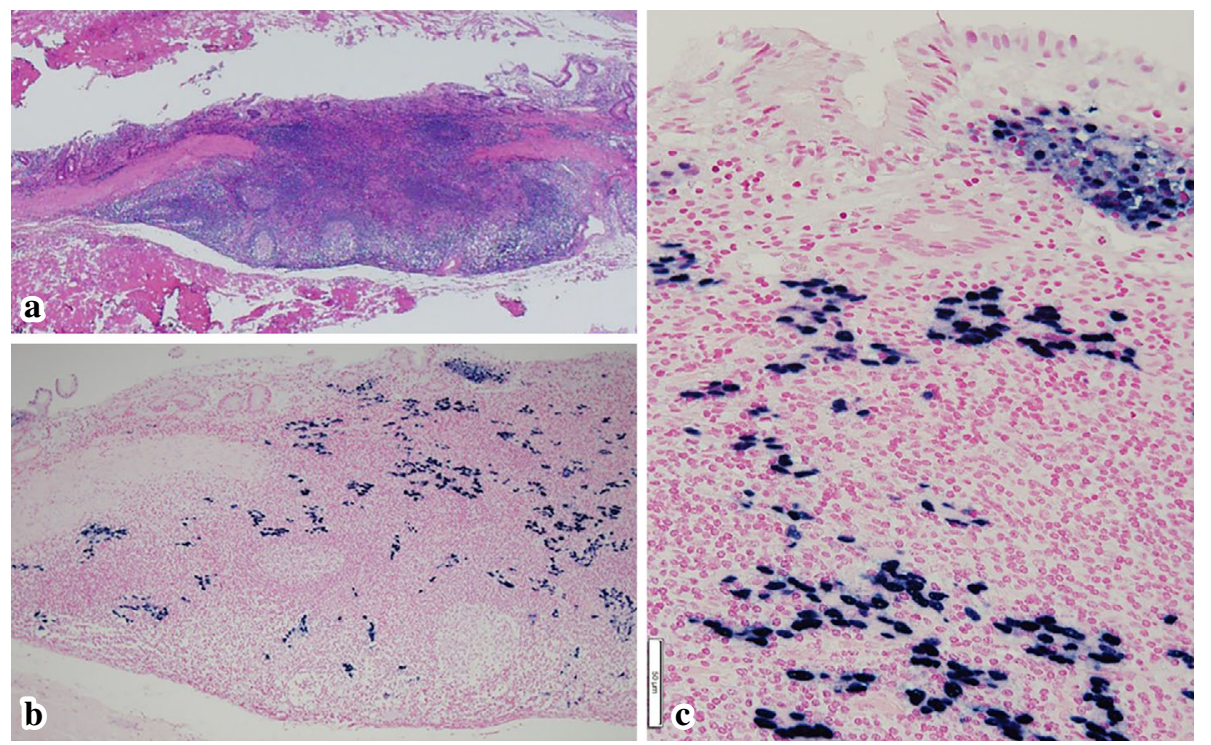

Figure 3. Pathological features of ESD specimen. a: Low-power microscopic picture of Hematoxylin and Eosin staining. The tumor was $10 \mathrm{~mm}$ in diameter and invaded $0.8 \mathrm{~mm}$ into the submucosal layer. The pathological type was CLS, and lymphovascular infiltration was negative. b: Middle-power microscopic picture of EBER1 ISH. c: High-power microscopic picture of EBER1 ISH. Almost all tumor cell nuclei were positive for EBER1 ISH. The lesion was confirmed to be EBV GC. 
cause of the risk of recurrence from possible LNM, we recommended he undergo an additional curative surgical operation with lymph node dissection based on the guidelines. At that time, we also informed him of the reported data concerning the relatively low risk of LNM from early EBV GC. The patient decided not to undergo the surgery or adjuvant chemotherapy. He has been in good health for over three years since ESD without disease recurrence and is being observed using endoscopy and CT.

\section{Discussion}

ESD is performed for the local treatment of GC lesions of the stomach, and the criteria for ESD are strictly limited to GC lesions with a very low risk of LNM. Ordinarily, poorly differentiated-type predominant GC with invasion into the submucosal layer has some risk of LNM and is indicated for surgical operation with regional lymph node dissection. Thus, since our present case with a GC lesion of poorly differentiated-type predominant GC with invasion into the submucosal layer was indicated for an additional surgical procedure, we recommended the patient undergo this procedure. However, he did not agree to it and remains in disease-free good health over three years after ESD.

Recently, accumulating data has shown that the risk of LNM from early EBV GC without local lymphovascular infiltration is low. Tokunaga et al. reported that there was no marked incidence of LNM among 75 EBV GC cases compared with 53 instances among 562 EBV-negative GC cases restricted to the mucosa and submucosal layer (7). Park et al. also reported that EBV positivity was a favorable risk factor for LNM in submucosa-invasive early GC (14). They suggested that an examination with EBER ISH should be considered for endoscopically resected specimens, especially in cases of submucosa-invasive early GC showing no lymph vessel invasion and clear resection margins. These reports indicate the possible further extension of the curative ESD criteria for early EBV GC invading into the submucosal layer without local lymphovascular infiltration. A precise examination using special staining is important for evaluating the lymphovascular infiltration of ESD specimens. Indeed, Lee et al. reported favorable outcomes in an early EBV GC case series treated with ESD (15).

In many cases, EBV GC is located in the upper part of the stomach and remnant stomach. Therefore, such extension of the criteria may prevent unnecessarily excessive surgery, such as total gastrectomy.

\section{Conclusions}

We herein reported a favorable clinical outcome for over three years of a case of early EBV GC invading the submucosal layer that was treated with ESD. Some cases of early EBV GC with invasion into the submucosal layer may be candidates for the further extension of the ESD criteria. The further accumulation of outcome data on early EBV GC treated with ESD is required in order to prevent possible overtreatment.

Informed consent was obtained from the patient.

The authors state that they have no Conflict of Interest (COI).

\section{References}

1. The Cancer, Genome Atlas, Research Network. Comprehensive molecular characterization of gastric adenocarcinoma. Nature 513: 202-209, 2014.

2. Murphy G, Pfeiffer R, Camargo MC, et al. Meta-analysis shows that prevalence of Epstein-Barr virus-positive gastric cancer differs based on sex and anatomic location. Gastroenterology 137: 824833, 2009.

3. Yanai H, Nishikawa J, Mizugaki Y, et al. Endoscopic and pathologic features of Epstein-Barr virus-associated gastric carcinoma. Gastrointest Endosc 45: 236-242, 1997.

4. Watanabe H, Enjouji M, Imai $T$, et al. Gastric carcinoma with lymphoid stroma: its morphologic characteristics and prognostic correlations. Cancer 38: 232-243, 1976.

5. Song HJ, Srivastava A, Lee J, et al. Host inflammatory response predicts survival of patients with Epstein-Barr virus-associated gastric carcinoma. Gastroenterology 139: 84-92, 2010.

6. Yanai H, Yahara N, Furuya T, et al. Long-term survival of patient with Epstein-Barr virus-positive gastric cancer treated with chemotherapy: case report. J Gastrointest Cancer 47: 107-110, 2016.

7. Tokunaga M, Land CE. Epstein-Barr virus involvement in gastric cancer: biomarker for lymph node metastasis. Cancer Epidemiol Biomarkers Prev 7: 449-450, 1998.

8. Bourke MJ, Neuhaus H, Bergman JJ. Endoscopic submucosal dissection: indications and application in western endoscopy practice. Gastroenterology 154: 1887-1900, 2018.

9. Japanese Gastric Cancer Association. Japanese gastric cancer treatment guidelines 2014 (ver. 4). Gastric Cancer 20: 1-19, 2017.

10. Hatta W, Gotoda T, Oyama $T$, et al. Is radical surgery necessary in all patients who do not meet the curative criteria for endoscopic submucosal dissection of early gastric cancer? A multi-center retrospective study in Japan. J Gastroenterol 52: 175-184, 2017.

11. Yanai H, Matsubara Y, Kawano T, et al. Clinical impact of strip biopsy for early gastric cancer. Gastrointest Endosc 60: 771-777, 2004.

12. Pyo JH, Lee H, Min BH, et al. Early gastric cancer with a mixedtype Lauren classification is more aggressive and exhibits greater lymph node metastasis. J Gastroenterol 52: 594-601, 2017.

13. Lim H, Lee IS, Lee JH, et al. Clinical application of early gastric carcinoma with lymphoid stroma based on lymph node metastasis status. Gastric Cancer 20: 793-801, 2017.

14. Park JH, Kim EK, Kim YH, et al. Epstein-Bar virus positivity, not mismatch repair-deficiency, is a favorable risk factor for lymph node metastasis in submucosa-invasive early gastric cancer. Gastric Cancer 19: 1041-1051, 2016.

15. Lee JY, Kim KM, Min BH, et al. Epstein-Barr virus-associated lymphoepithelioma-like early gastric carcinomas and endoscopic submucosal dissection: Case series. World J Gastroenterol 20: 1365-1370, 2014.

The Internal Medicine is an Open Access journal distributed under the Creative Commons Attribution-NonCommercial-NoDerivatives 4.0 International License. To view the details of this license, please visit (https://creativecommons.org/licenses/ by-nc-nd/4.0/).

(C) 2019 The Japanese Society of Internal Medicine

Intern Med 58: 3247-3250, 2019 